\title{
Resolving the controversy of the proportion validity effect: Volitional attention is not required, but may have an effect
}

\author{
Sophie N. Lanthier ${ }^{1}$ - David W.-L. Wu ${ }^{1}$. Craig S. Chapman ${ }^{2} \cdot$ Alan Kingstone $^{1}$
}

Published online: 16 July 2015

(C) The Psychonomic Society, Inc. 2015

\begin{abstract}
Response time (RT) is facilitated when a target appears at a cued (valid) location versus an uncued (invalid) location. Interestingly, this valid-versus-invalid RT difference increases as the percentage of valid trials increases. In the present study, we investigated the mechanism responsible for this proportion valid cueing effect (PVE). The PVE is thought to reflect changes in voluntary attentional allocation, with greater attention being committed endogenously to the cued location as the percentage of valid trials increases. However, recent research has suggested that the PVE may reflect a form of implicit learning between the cue and the target location that is developed outside of awareness, and that this determines how attention is allocated. This lack of convergence may be due to methodological differences in how voluntary processing has been inferred. To test this issue, we generated a method that would allow the measurement of different degrees of volitional attention. In addition, we manipulated whether participants were instructed to attend to the cue-target relationship and determined whether this explicit engagement of attention influenced the PVE. We found that for both peripheral and central cues, volitional control is not required for a PVE; however, volitional control can modulate a PVE that is produced by central cues. Thus, a PVE is not a reliable indicator of volitional control, but its sensitivity to volitional control varies across cues. The present data shed light on the mechanism subserving the PVE and lend support
\end{abstract}

Sophie N. Lanthier

snlanthier@gmail.com

1 University of British Columbia, Vancouver, British Columbia, Canada

2 University of Alberta, Edmonton, Alberta, Canada to the theory that different cues engage, to some degree, qualitatively different forms of visuospatial attention.

Keywords Space-based attention · Attention . Executive control

When attention is directed to an upcoming target location by a valid cue, the response time (RT) to the target is faster than when attention is misdirected to a nontarget location by an invalid cue. One of the most robust findings in the covertorienting literature is that this cueing effect (valid $\mathrm{RT}<$ invalid $\mathrm{RT}$ ) increases in magnitude as the percentage of valid trials increases (e.g., Kingstone, 1992). This proportion valid cueing effect (PVE) is believed to reflect the involvement of volitional control of visual attention. Specifically, the more a cue predicts the upcoming target location, the more individuals are thought to allocate attention volitionally to the cued location. However, Risko and Stolz (2010b) suggested that the PVE might not reflect volitional orienting. In their study, participants were unable to correctly report changes in cue validity, and yet a PVE was still observed. The implication here is that the PVE reflects implicit learning of cue-target contingencies that occurs outside of awareness.

There is certainly research to support an implicit-learning account. For instance, Chun and Jiang $(1998,1999)$ have shown that visual attention can be guided by contextual information that participants acquire unknowingly. Similarly, in a cueing paradigm, Lambert and colleagues (Lambert, Naikar, McLahan, \& Aitken, 1999; Lambert \& Sumich, 1996; Shin, Marrett, \& Lambert, 2011) reported that if participants are briefly presented with letter cues that predicted the particular location of an upcoming target, they showed cueing effects despite being unable to describe the cue-target relations, or 
even to acknowledge seeing the cues. These, and similar studies, suggest that conscious awareness of the cue (Kentridge, Heywood, \& Weiskrantz, 1999; Lambert et al., 1999; Lambert \& Sumich, 1996; McCormick, 1997) and/or of the relationship between the cue and the target (López-Ramón et al., 2011; Risko \& Stolz, 2010b) is not necessary for orienting of spatial attention to occur.

There is some debate, however, regarding the extent to which the PVE is driven by implicit processes. Some research has suggested that implicit processing can contribute to the PVE, but only when the cues are presented briefly in the periphery (i.e., when attention is engaged automatically; Bartolomeo, Decaix, \& Siéroff, 2007; Lambert \& Holmes, 2004; Lambert et al., 1999; Lambert \& Sumich, 1996). For instance, Bartolomeo, Decaix, and Siéroff had participants perform cueing experiments with different percentages of valid trials. Each experiment began with a block of 24 trials in which $50 \%$ of the trials were valid, followed by a second block of 90 trials in which either $20 \%, 50 \%$, or $80 \%$ of the trials were valid. After each experiment, participants reported (1) whether a relationship existed between the cue and the target and (2)whether the cues predicted the target or a nontarget location. On the basis of their answers to these questions, participants were divided into two groups: those who could detect the nature of the cue-target relation and those who could not. Consistent with an implicit-learning account, the PVE elicited by peripheral onsets occurred regardless of whether or not participants could detect the cue-target relationship. However, for central symbolic cues, a PVE was observed solely for those participants who could detect the changes in cue-target contingency. Accordingly, Bartolomeo and colleagues suggested that implicit learning provides an account of the PVE with automatic orienting to peripheral cues, but volitional orienting is critical to the PVE for central cues. This distinction converges with a body of evidence that peripheral and central cues engage qualitatively distinct attentional processes (Jonides, 1981; Posner, 1980; Taylor \& Klein, 1998; Theeuwes, 1991; Tipples, 2008; Vecera \& Rizzo, 2006; Yantis \& Jonides, 1990; see also Chica, Bartolomeo, \& Lupiáñez, 2013, for a review; and Funes, Lupiáñez, \& Milliken, 2007, for a double dissociation).

However, the notion that the PVEs for peripheral and central cues are driven by different processes was bought into question by Risko and Stolz (2010b). They ran a study similar to Bartolomeo et al. (2007), but rather than asking participants merely to detect the general contingency between the cue and target (i.e., was the contingency predictive or not?), they asked participants to generate a specific value that characterized the contingency between the cue and the target. Like Bartolomeo et al., the cue-target relation was either chance $(50 \%)$ or predictive (75\% valid in the Risko \& Stolz, 2010b, study). Risko and Stolz (2010b) found that the PVE for peripheral cues was unrelated to participants' estimates of the cue-target relationship. This provided a conceptual replication of the Bartolomeo et al. finding. However, Risko and Stolz (2010b) also found that the PVE for central cues was unrelated to participants' estimates of the cue-target relationship, which diverged from Bartolomeo et al.'s report that the PVE produced by central cues emerged only for participants who were aware of the cue-target relation. In sum, both Bartolomeo et al. and Risko and Stolz (2010b) found that for peripheral cues the PVE is driven by implicit processes. However, the two studies disagreed regarding whether the PVE for central cues is driven by implicit processes. Bartolomeo et al. suggested that for central cues the PVE reflects volitional control processes, whereas Risko and Stolz (2010b) suggested that the PVE for central cues is implicit in nature (see also Chica \& Bartolomeo, 2010, and Risko \& Stolz, 2010a, for their commentaries on this matter).

Several accounts are possible of why the central cue data for the two studies appear to conflict. Methodologically, there were fundamental differences between the studies, including, but not limited to, the fact that Risko and Stolz (2010b) did not include a low-validity condition (e.g., 25\%), which would have exposed participants in the Bartolomeo et al. (2007) study to a broader range of cue-target contingencies, and doing so may have facilitated the participants' sensitivity to changes in cue-target contingencies (see below for a fuller discussion of this matter). The present article will include the full range of validity values.

Far more important, perhaps, is the fact that the studies differed in how they measured cue-target awareness in order to infer implicit processing. Certainly, the two studies shared the conceptual approach that a failure to accurately judge a cue-target contingency would suggest a lack of cue-target awareness and support the possibility that a PVE was driven by implicit processing. But within that framework, they differed in the types of opportunities for failure that they provided to participants.

In Risko and Stolz (2010b), the likelihood of an incorrect judgment was very high, because participants were required to generate the precise value characterizing the cue-target contingency, a value that could range from $0 \%$ to $100 \% .{ }^{1}$ In

${ }^{1}$ It should be noted that in principle Risko and Stolz (2010b) were not interested in the exact value given, per se, but rather in whether the value, whatever it was, correlated with the magnitude of the cueing effect. Moreover, one could argue that there is more power to detect a significant correlation with a continuous than with a dichotomous variable. Nevertheless, these potential benefits may have been undermined by the facts that (1)participants were inaccurate and variable in their estimates and (2)cue-target contingency was varied between groups, and as a result, participants did not have the opportunity to sample the range and relative change in cue-target contingencies. 
Bartolomeo et al. (2007), participants were grounded on a chance $(50 \%)$ cue-target contingency and were merely asked to detect whether or not the current cue-target contingency violated that contingency (yes or no) and, if participants indicated "yes," whether the cue was usually valid or invalid.

In short, Risko and Stolz's (2010b) and Bartolomeo et al.'s (2007) conflicting results might have been driven, at least in part, by the use of different subjective reports of awareness that required very different degrees of precision when participants reported the cue-target contingencies - that is, a fine level of precision (Risko \& Stolz, 2010b) or a coarse level of precision (Bartolomeo et al., 2007). As such, the different approaches may have measured different levels of awareness of the cue-target contingency, with a coarse/general level being critical to the PVE and a fine/precise level not being critical. This gives rise to the possibility that the level of awareness that Bartolomeo and colleagues suggested is required to produce a PVE was present, but not captured, by the measure used by Risko and Stolz (2010b). Or, to put it differently, whereas Risko and Stolz (2010b) demonstrated that participants did not have a precise understanding of the cue-target contingency, it is possible that their participants nonetheless acquired the general sense of the cue-target contingency that Bartolomeo and colleagues considered essential for producing the PVE.

The differences in awareness reported in these studies may have been accentuated further by the fact that the participants had different opportunities to understand the cue-target contingencies over the course of the experiment. Risko and Stolz (2010b) manipulated cue validity between participants, which may have made understanding and accurately reporting the cue-target contingency more challenging, because the participants were unable to experience and contrast the high- and low-validity conditions during the experiment. In contrast, Bartolomeo et al. (2007) ran the high- and low-cue-validity conditions within participants, which allowed participants to experience both the high- and low-validity conditions, and thus to compare their experiences in both conditions to further inform their reports of cue utility.

In the present study, as in the two key investigations reviewed above, we adopted the conceptual framework that a failure to accurately report the cue-target relation may indicate that participants are unaware of the cue-target contingency and that implicit processes are in play. However, we also adopted the working hypothesis that the different approaches for measuring awareness of cue-target contingencies may tap into different levels of awareness - general or precise-with only a general level of awareness of the cue-target contingency being relevant to the PVE.

To test this hypothesis, we had participants generate a precise estimate for the cue-target contingencies (in keeping with Risko \& Stolz, 2010b), but introduced a within-participants design (in keeping with Bartolomeo et al., 2007). By combining these two designs, we could extract a relative change in the precise estimates and determine how the relative change in these estimates related to the PVE. This relative change served as a measure of participants' coarse awareness of changes in the cue-target contingencies. Of course, by extracting precise estimates from the participants, we could also directly test, and seek to replicate, the findings of Risko and Stolz (2010b), but now within the context of a withinparticipants design.

Finally, as an additional manipulation, we introduced a condition whereby participants were asked explicitly to pay attention to the cue-target contingencies. Note that instructing participants simply to attend to the cue-target contingencies should not affect implicit processes, since these processes are understood to work independently of (or in parallel with) volitional attentional processes (Cleeremans, Destrebecqz, \& Boyer, 1998). However, being instructed of the cue-target relations could create a top-down task set that would enhance the process of becoming aware of cue-target contingencies. As such, in the present study we not only measured implicit processes by failures to accurately report cue-target contingencies, but also introduced a method to assess directly the role of volitional control in the PVE. Using instructions avoids relying exclusively on posttest self-reports and the weaknesses associated with this technique (see Vaquero, Fiacconi, \& Milliken, 2010, for a more thorough discussion on this topic), such as a lack of precision regarding when awareness regarding a cue-target contingency emerged during testing.

\section{Experiment 1}

Experiment 1 extended the Risko and Stolz (2010b) investigation, in which participants were required to perform a standard cueing task in which peripheral rectangle cues were used to direct attention. In addition to manipulating cue validity, we explicitly instructed half of the participants to pay attention to the relation between the cues and spatial locations, to enhance the process of their becoming aware of cue-target contingencies. An implicit-processing account predicts that the PVE should be independent of awareness as indexed by subjective reports, and should be unaffected by the instruction manipulation. A purely volitional account predicts that the PVE should only occur if participants are instructed to pay attention to the cue-target contingencies or (previous limitations of the subjective report method notwithstanding) if participants become aware of the cue-target contingencies, as indexed by subjective reports.

To further provide the opportunity for endogenous orienting to impact the PVE, we extended the cue-target stimulus onset asynchronies (SOA) that had been used by Risko and Stolz (2010b) from 150 to $350 \mathrm{~ms}$ (the SOA that was used for their central cue). Risko and Stolz (2010b) had used the 
shorter SOA for the peripheral cue to try to engage rapid exogenous orienting while preventing the involvement of any endogenous orienting. By lengthening the SOA in the present study, and thereby providing the opportunity for endogenous processes to impact the PVE, the present study provides a strong test of the volitional account. Furthermore, by keeping the SOA fixed at $350 \mathrm{~ms}$ in both experiments, we avoided confounding cue type with SOA, and thus ensured that any performance change between experiments could be attributed to attentional processes related to cue type (see López-Ramón, Chica, Bartolomeo, \& Lupiáñez, 2011, for data from a similar experiment)

\section{Method}

Participants A total of 80 participants with normal or corrected-to-normal vision were either given credit or paid $\$ 10$ for their time.

Design The study used a 2 (Instruction: instructed and not instructed $) \times 2$ (Cue Validity: valid and invalid $) \times 3$ (Percentage Valid: $25 \%, 50 \%$, and $75 \%$ ) mixed design in which instruction was manipulated between participants and cue validity and percentage valid were manipulated within participants.

Apparatus E-Prime 2.0 controlled the timing and presentation of the stimuli and logged response accuracy and RTs. Stimuli were presented on standard 15-in. CRT monitors with a resolution of $1,024 \times 728$. Participants viewed the monitor unrestrained at a distance of approximately $60 \mathrm{~cm}$.

Stimuli The stimulus display consisted of a central fixation cross that measured $0.6 \mathrm{~cm}^{2}$. The cues were solid rectangles ( $1.0 \mathrm{~cm}$ vertical, $1.4 \mathrm{~cm}$ horizontal) displayed $4.2 \mathrm{~cm}$ above or below fixation. The targets were either "\%" or "\#" signs measuring $0.9 \mathrm{~cm}$ vertically and $0.6 \mathrm{~cm}$ horizontally. The targets appeared $3.0 \mathrm{~cm}$ above or below fixation equally often. All stimuli were white and were presented on a black background.

\section{Procedure}

Following Risko and Stolz (2010b), all participants were asked to indicate by buttonpress (either " $\mathrm{c}$ " or " $\mathrm{m}$ ") whether a target was "\%" or "\#." We stressed that participants should try to keep their eyes at fixation at all times, and that both speed and accuracy were equally important in responding. The only procedural difference between participants was the instructions. The participants in the instructed condition were instructed to try to determine the different relationships that existed between the rectangle cues and targets in each block (e.g., in some blocks the cue would indicate the correct location of the upcoming targets more than in others). The participants in the not-instructed condition did not receive any such instruction prior to beginning the task. Half of the participants were assigned to the instructed condition and the other half to the not-instructed condition.

Each trial began with the fixation cross for $500 \mathrm{~ms}$, followed by the onset of a cue either above or below the cross for $50 \mathrm{~ms}$. After cue offset, there was a 300 -ms delay before the target was presented. A response extinguished the display for $500 \mathrm{~ms}$ before the next trial began. See Fig. 1 for an example of the trial sequences for valid and invalid cues. Participants performed 16 practice trials to get acquainted with the task. The experiment consisted of three blocks of 416 trials, ${ }^{2}$ with the percentage of valid cues in a given block being $25 \%, 50 \%$, or $75 \%$. In all conditions and both experiments, the term valid refers to a spatial correspondence between a cue and target (e.g., cue left-target left), regardless of the cue predictability. The order of the blocks was counterbalanced across participants. After each block, participants were asked to fill out a questionnaire that asked them (1)to indicate whether the target appeared more frequently in the same location as the "white rectangle" (i.e., the cue) or in a different location from the "white rectangle," and (2)to write down the percentage of trials on which the "white rectangle" (i.e., the cue) indicated the correct location of the upcoming target. The experiment took approximately $60 \mathrm{~min}$ in total.

\section{Results}

Data-trimming procedure Prior to the RT and error analysis, outliers were removed using a modified recursive trimming procedure (see Van Selst \& Jolicœur, 1994, for a justification). Using this procedure, data are trimmed over several cycles by using a unique cutoff criterion (i.e., a certain number of standard deviations away from the mean) that is set independently during each cycle, for each participant, in each condition. The value of the cutoff is dynamically altered on the basis of the sample size in each cycle of the procedure. This data-trimming procedure resulted in $4.3 \%$ of the RT data being discarded. Mean correct RTs and errors were analyzed using a three-way

\footnotetext{
${ }^{2}$ When cues are informative (e.g., indicate a valid location more than $50 \%$ of the time), participants should learn the cue-target associations, whether by volitional or implicit means (Chun \& Jiang, 1998, 1999), if they are given enough time. This assumption leads to questions about whether the participants in Risko and Stolz's (2010b) study had enough time (they were provided only 192 trials in $10 \mathrm{~min}$ ) to volitionally or implicitly learn the cue-target associations. Indeed, Risko and Stolz (2010b) have been criticized for potentially creating experimental conditions that stressed automatic processes (Chica \& Bartolomeo 2010). We increased the number of trials in order to provide participants with more of an opportunity to experience the cue-target relations.
} 


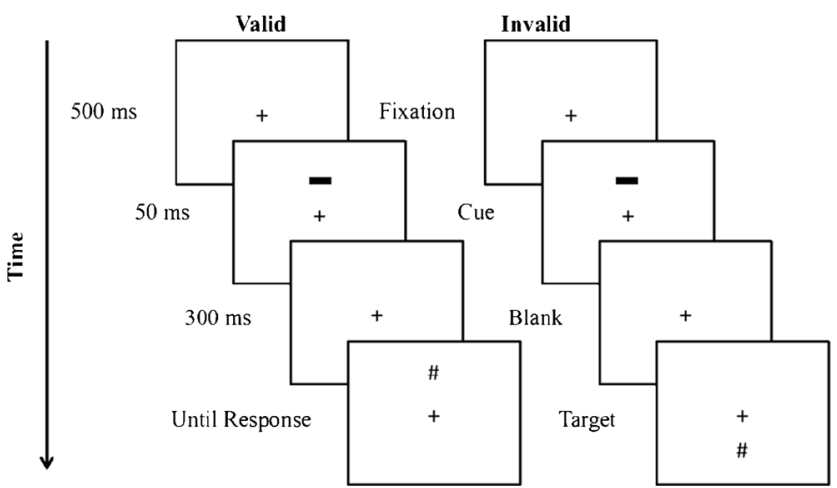

Fig. 1 Example of the trial sequences of a valid and an invalid trial in Experiment 1. Note that in the actual experiment, the contrast was reversed (i.e., black background and white stimuli)

mixed analysis of variance (ANOVA) with Instruction (two levels: instructed and not instructed) as a between-participants factor and Cue Validity (two levels: valid and invalid) and Percentage Valid (three levels: $25 \%$ valid, $50 \%$ valid, and $75 \%$ valid) as within-participants factors. For all analyses in all experiments, if Mauchly's test of sphericity was significant $(p<.25)$, the relevant degrees of freedom were adjusted using the Greenhouse-Geisser (if $\varepsilon \leq .70$ ) or Huynh-Feldt (if $\varepsilon>$ .70) method. Furthermore, it should be noted that although all of the analyses reported below collapse across blocks, we also conducted all of the analyses with Block as a factor; for example, if endogenous processes impact the PVE, one might expect participants in the instructed condition to discover the cue-target relationship before the not-instructed participants. Block never interacted with instruction, cue validity, or percentage valid (all $p \mathrm{~s}>.2$ ). ${ }^{3}$ Mean RTs, percentage errors, and cueing effects are presented in Table 1.

Response times We observed a main effect of cue validity $[F(1,78)=63.05, M S E=701.66, p<.001]$, such that responses were quicker to validly cued targets $(524 \mathrm{~ms})$ than to invalidly cued targets $(543 \mathrm{~ms})$. There was also a significant interaction between percentage valid and cue validity $[F(2$, $156)=27.79, M S E=391.47, p<.001]$, such that the effect of cue validity increased as the percentage of valid trials increased: This is the PVE. No other main effects or interactions were significant (all $F \mathrm{~s}<2.2$ ).

To further assess the interaction between cue validity and percentage valid, the magnitude of the cueing effect was determined by subtracting the RTs in the valid condition from the RTs in the invalid condition. Three two-tailed repeated

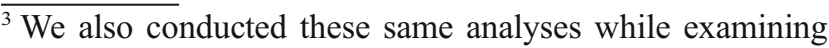
only the first block of trials of each participant. Note that Awareness and Percentage Valid are now both betweenparticipants factors, and Cue Validity is a within-participants factor. These findings mirror those reported in full in the Results.
}

Table 1 Mean response times (in milliseconds) and percentage errors in Experiment 1 as a function of instruction, percentage of valid trials, and cue validity

\begin{tabular}{lllllll}
\hline \multicolumn{7}{c}{ Percentage of Valid Trials } \\
& \multicolumn{7}{c}{ Response Times } & \multicolumn{4}{l}{ Percentage Error } \\
Cue Awareness & $25 \%$ & $50 \%$ & $75 \%$ & $25 \%$ & $50 \%$ & $75 \%$ \\
Instructed & & & & & & \\
$\quad$ Valid & 521 & 526 & 522 & 6.8 & 7.6 & 6.9 \\
$\quad$ Invalid & 520 & 541 & 563 & 6.9 & 7.7 & 7.2 \\
$\quad$ Difference & -1 & 15 & 41 & 0.1 & 0.1 & 0.3 \\
Not Instructed & & & & & & \\
$\quad$ Valid & 521 & 546 & 509 & 6.1 & 5.5 & 5.5 \\
$\quad$ Invalid & 531 & 562 & 543 & 5.2 & 5.5 & 5.8 \\
$\quad$ Difference & 10 & 16 & 34 & -0.9 & 0.0 & 0.3 \\
\hline
\end{tabular}

measures $t$ tests (which, after applying a Bonferroni correction for multiple comparisons, had a family-wise error rate of .05) showed that cueing effects were larger in the $75 \%$-valid condition $(37 \mathrm{~ms})$ than in both the $50 \%$-valid condition ( $16 \mathrm{~ms})$ $[t(79)=4.62, p<.001]$ and the $25 \%$-valid condition $(15 \mathrm{~ms})$ $[t(79)=6.87, p<.001]$, and also were larger in the $50 \%$-valid condition than in the $25 \%$-valid condition $[t(79)=2.75, p<.008]$.

Errors No main effects or interactions were significant (all $F_{\mathrm{S}}<2.8$ ).

Participant estimates Participants' estimates of the percentages of valid trials in each block were analyzed using a twoway ANOVA with Instruction (two levels: instructed and not instructed) as a between-participants factor and Percentage Valid (three levels: $25 \%, 50 \%, 75 \%$ ) as a within-participants factor. The mean participant estimates are shown in Fig. 2.

A main effect of percentage valid emerged $[F(1.69$, $133.37)=39.30, M S E=379.47, p<.001]$, such that participants' estimates of the percentage of valid trials increased as the percentage of valid trials increased. No other main effects or interactions were significant (all $F_{\mathrm{S}}<2.5$ ).

To further assess the main effect of percentage valid, three two-tailed repeated measures $t$ tests revealed that the estimates were significantly higher in the $75 \%$-valid condition $(65 \%)$ than in both the $50 \%$-valid condition $(54 \%)[t(79)=4.34, p<.001]$ and the $25 \%$-valid condition $(40 \%)[t(79)=7.24, p<.001]$. Estimates were also higher in the $50 \%$-valid condition than in the $25 \%$-valid condition [t(79) $=5.77, p<.001]$.

Additionally, one-sample $t$ tests revealed that the estimates for the $25 \%$-valid block were significantly higher than $25 \%$ $[t(79)=6.25, p<.001]$, the estimates for the $50 \%$-valid block were significantly higher than $50 \%[t(79)=2.52, p<.05]$, and the estimates for the $75 \%$-valid block were significantly lower than $75 \%[t(79)=4.91, p<.001]$. 


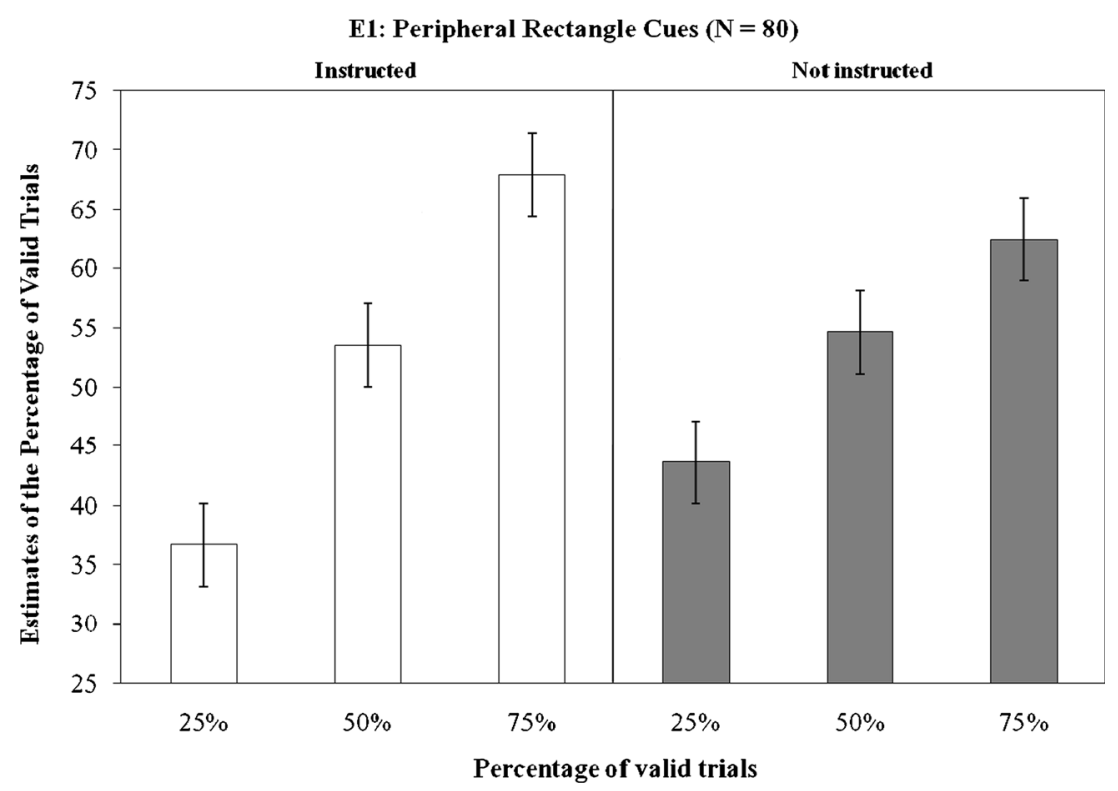

Fig. 2 Participant estimates of the percentages of valid trials as a function of instruction (instructed vs. not instructed) and percentage valid (25\% vs. $50 \%$ vs. 75\%) in Experiment 1. Error bars represent 95\% confidence intervals, as defined by Masson and Loftus (2003)

Relation between participant-specific estimates and performance A Pearson correlation analysis revealed that the participant estimates and the magnitudes of their cueing effects were not significantly correlated in either RTs or errors in the $25 \%$ [RT, $r(80)=.17, p=.13$; errors, $r(80)=-.07, p=052], 50 \%[\mathrm{RT}$, $r(80)=-.14, p=.21$; errors, $r(80)=-.02, p=.84]$, or $75 \%[\mathrm{RT}$, $r(80)=.13, p=.24$; errors, $r(80)=-.18, p=.11]$ valid conditions. Furthermore, the same analysis was conducted on each instruction condition separately and revealed no significant correlations.

Relationship between the general change in participant estimates and cueing effects as a function of percentage of valid trials We calculated the slopes relating the participants' estimates of the numbers of valid trials and the magnitudes of their cueing effects with respect to the percentage of valid trials (i.e., $25 \%, 50 \%$, or $75 \%$ ) for each participant, in both the instructed and uninstructed conditions separately. Next, we correlated these measures to examine the relationship between the change in general awareness of cue utility (i.e., the change in the participant estimates as a function of percentage valid) and the PVE (i.e., the change in the magnitude of cueing effects with change in the percentage of valid trials). We found no correlation between the change in participants' estimates and the change in the magnitudes of their cueing effects as a function of the percentage of valid trials, regardless of whether participants were instructed, $r(40)=.14, p=.40$, or not, $r(40)=.11, p=.52$. The correlation is plotted in Fig. 3.

\section{Discussion}

In Experiment 1, the magnitude of the cueing effect increased as the percentage of valid trials increased (the PVE).
Specifically, cueing effects were largest when $75 \%$ of the trials were valid, and smallest when $25 \%$ of the trials were valid. In addition, participants underestimated the actual percentage valid in the $75 \%$-valid condition, overestimated the actual percentage valid in the $50 \%$-valid condition, and overestimated the actual percentage valid in the $25 \%$-valid condition. Critically, in Experiment 1, participants' awareness of the cue-target relations had no influence on the PVE or on their estimates of the percentage of valid trials.

Finding that participants' specific estimates of the percentage of valid peripheral cues do not predict the magnitude of the RT cueing effect indicates that a precise level of awareness of the cue-target contingency is not crucial to the PVE. This replicates Risko and Stolz (2010b). Furthermore, the failure to find a relationship between the relative change in participants' estimates and the change in the magnitude of cueing effects demonstrates that a general awareness of changes in the cuetarget contingency is also not critical to the PVE. This replicates Bartolomeo et al. (2007).

Collectively, the results of Experiment 1 confirm that the PVE for peripheral cues occurs regardless of participants' specific or general awareness of cue-target contingencies. These data suggest that the PVE reflects implicit processes rather than explicit awareness of the cue-target relationship. This conclusion is reinforced by our finding that the PVE is insensitive to whether or not participants are explicitly instructed to pay attention to the cue-target relationship.

Finally, note that our conclusion is not that top-down processes are unable to modulate the effect of a nonpredictive peripheral cue, but rather that the PVE-produced by variation in cue-target validities - is driven by implicit processes. The former point, that top-down processes can impact 


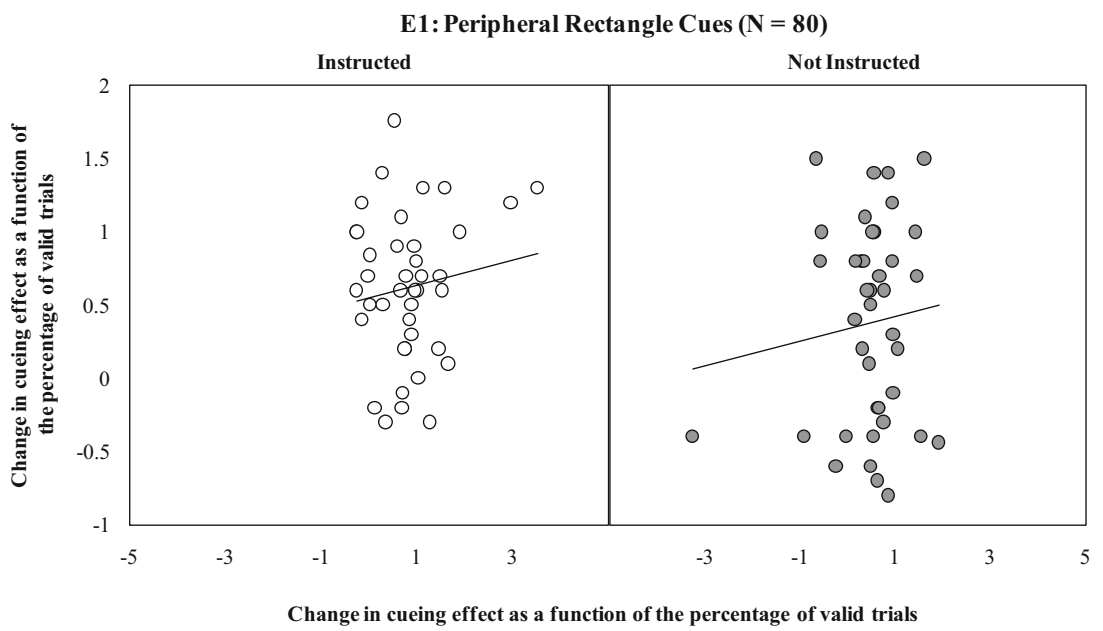

Fig. 3 The correlation between the change in participants' estimates (percentages) and the change in the magnitude of their cueing effects (in milliseconds) as a function of the percentage of valid trials, for each instruction condition (instructed vs. not instructed) in Experiment 1

a nonpredictive peripheral cue, has been made frequently in the past, both with regard to the early facilitation effect of a peripheral cue (e.g., López-Ramón et al., 2011) and the subsequent emergence of a reverse cueing effect, called inhibition of return (e.g., Tipper \& Kingstone, 2005). For instance, López-Ramón et al. (2011) suggested that uninstructed participants who attempt to determine the relationship between a nonpredictive (50\% valid) peripheral cue and target (those participants who they call "good estimators") may modulate (in this case, reduce) the facilitatory effect of a peripheral cue.

\section{Experiment 2}

In Experiment 2, we used a central arrow cue in place of the peripheral rectangle cue used in Experiment1. Central cues also demonstrate a PVE (Bartolomeo et al. 2007; Risko \& Stolz, 2010b). Thus, as in Experiment1, we could assess whether awareness of cue-target relations influences the emergence of the PVE. With the exception of change in the attentional cue, all other aspects of Experiment 2 were the same as in Experiment 1.

\section{Method}

Participants A total of 80 new participants from the University of British Columbia were either given credit or paid $\$ 10$ for their time.

Design, apparatus, and procedure The design, apparatus, stimuli, and procedure were the same ones used in Experiment 1, except that the cues were now centrally located arrows $(0.8 \mathrm{~cm}$ vertical, $1.1 \mathrm{~cm}$ horizontal) that pointed up or down. Note that the arrow cues appeared for $300 \mathrm{~ms}$, and then the target was presented $50 \mathrm{~ms}$ later, thereby keeping the SOAs identical across experiments (i.e., $350 \mathrm{~ms}$ ).

\section{Results}

Our data analysis followed the same procedure that was used in the previous experiment, which resulted in $5.07 \%$ of the RT data being discarded. We also performed all of the analyses below with Block as a factor. As before, block did not interact with instruction, cue validity, or percentage valid (all $p \mathrm{~s}>.15){ }^{4}$ The mean correct RTs and cueing effects are presented in Table 2.

Response time A main effect of instructions was apparent $[F(1,78)=17.56, M S E=40,431.29, p<.001]$, such that responses were faster when participants were not instructed $(503 \mathrm{~ms})$ than when they were instructed $(580 \mathrm{~ms})$ about the cue-target relations. We also found a main effect of cue validity $[F(1,78)=21.193, M S E=2,862.549, p<.001]$, such that responses were faster to validly cued targets $(530 \mathrm{~ms})$ than to invalidly cued targets $(552 \mathrm{~ms})$. Additionally, the interaction between cue validity and percentage valid was significant $[F(1.51,117.48)=11.76, M S E=2,205.25, p<.001]$, such that the effect of cue validity increased as the percentage of valid trials increased: This is the PVE. Likewise, the interaction

\footnotetext{
${ }^{4}$ As before, we also conducted these same analyses while examining only the first block of trials of each participant. Our results mirrored those we report, with the exception that due to the loss of statistical power, the interaction between cue validity, percentage valid, and instruction was marginally significant, $p<.06$. Most critically, when participants were instructed, the interaction was significant $(p<.05)$, but not when participants were uninstructed $(p=.67)$.
} 
Table 2 Mean response times (in milliseconds) and percentage errors in Experiment 2 as a function of instruction, percentage of valid trials, and cue validity

\begin{tabular}{lllllll}
\hline \multicolumn{7}{c}{ Percentage of Valid Trials } \\
\cline { 2 - 7 } & \multicolumn{2}{l}{ Response Times } & \multicolumn{4}{l}{ Percentage Error } \\
\hline $\begin{array}{l}\text { Cue Awareness } \\
\text { Instructed }\end{array}$ & $25 \%$ & $50 \%$ & $75 \%$ & $25 \%$ & $50 \%$ & $75 \%$ \\
$\quad$ Valid & 564 & 558 & 566 & 6.7 & 6.5 & 7.2 \\
$\quad$ Invalid & 566 & 587 & 636 & 7.2 & 7.0 & 7.4 \\
$\quad$ Difference & 2 & 28 & 70 & 0.5 & 0.5 & 0.2 \\
Not Instructed & & & & & & \\
$\quad$ Valid & 498 & 499 & 494 & 7.7 & 7.3 & 6.8 \\
$\quad$ Invalid & 500 & 509 & 516 & 7.1 & 8.6 & 7.6 \\
$\quad$ Difference & 2 & 10 & 22 & -0.6 & 1.3 & 0.8 \\
\hline
\end{tabular}

between instruction and cue validity was significant $[F(1,78)$ $=4.99, M S E=2,862.55, p<.03]$, such that the effect of cue validity was larger when participants were instructed to attend to the cue-target relation. Finally, there was a significant three-way interaction between instruction, cue validity, and percentage valid $[F(1.51,117.48)=3.42, M S E=2,205.25, p$ $<.05$ ], reflecting that the PVE in the instructed condition was magnified at the $75 \%$ and $50 \%$ validities, but was equal to that in the not-instructed condition at $25 \%$ (see Table 2). No other main effects or interactions were significant (all $F_{\mathrm{S}}<1.9$ ).

As in Experiment 1, two-tailed repeated measures $t$ tests were applied to the cueing effects. In the instructed condition, cueing effects were larger in the $75 \%$-valid condition (70 ms) than in the $25 \%$-valid condition $(2 \mathrm{~ms})[t(39)=3.16, p<.003]$ and the $50 \%$-valid condition (28 ms) $[t(39)=2.45, p<.02]$. Also, cueing effects were larger in the $50 \%$-valid condition than in the $25 \%$-valid condition $[t(39)=2.34, p<.03]$. When participants were not instructed, the cueing effect was larger in the $75 \%$-valid condition ( $22 \mathrm{~ms}$ ) than in the $25 \%$-valid condition $(2 \mathrm{~ms})[t(39)=2.86, p<.008]$. No other comparisons were significant. Thus, whereas the PVE occurred whether or not participants were instructed to attend to the cue-target relation, the PVE was magnified when participants' attention was directed to the cue-target relations. This effect of instruction lends support to the idea that a volitional component can influence the nature of the PVE.

Error No main effects or interactions were significant (all $F_{\mathrm{s}}<1.9$ ).

Participant estimates Participants' estimates of the percentage valid in each block were analyzed as a function of instruction and percentage valid. The mean participant estimates are shown in Fig. 4. The ANOVA revealed a main effect of percentage valid $[F(1.506,156)=40.52, M S E=248.27, p<$
.001], such that participants' estimates of the percentage of valid trials increased as the percentage of valid trials increased. No other effects were significant (all $F \mathrm{~s}<1.2$ ). Three twotailed repeated measures $t$ tests revealed that estimates were significantly higher in the $75 \%$-valid condition $(64 \%)$ than in both the $25 \%$-valid condition $(42 \%)[t(79)=8.19, p<$ $.001]$ and the $50 \%$-valid condition $(52 \%)[t(79)=5.69, p<$ $.001]$. Estimates were also higher in the $50 \%$-valid condition than in the $25 \%$-valid condition $[t(79)=3.95, p<.001]$.

Additionally, two-tailed one-sample $t$ tests showed that estimates for the $25 \%$-valid block were significantly higher than $25 \%[t(79)=7.44, p<.001]$, estimates for the $50 \%$-valid block were not different from $50 \%[t(79)=0.99, p=.33]$, and estimates after the $75 \%$-valid block were significantly lower than $75 \%[t(79)=7.36, p<.001]$.

Relation between participant-specific estimates and performance A Pearson correlation analysis revealed no significant correlations between the participants' estimates and the magnitudes of their cueing effects (on RTs), save for the lone exception of the $25 \%$-valid condition $(r=-.25, p<.02)$ in which participants with lower estimates had larger cueing effects (although, overall, this cueing effect was very small; i.e., $2 \mathrm{~ms}$ ). Furthermore, when the same analysis was conducted on each instruction condition separately, no correlations were significant save for the $25 \%$-valid instructed condition $(r=$ $-.38, p<.02$; and not in the $25 \%$-valid not-instructed condition: $r=-.10, p=.54)$.

Relationship between the general change in participant estimates and cueing effects as a function of the percentage of valid trials As in Experiment1, we calculated the slope relating the change in the participants' estimates and the change in the magnitude of their cueing effects with respect to the percentage of valid trials (i.e., $25 \%, 50 \%$, and $75 \%$ ) for both the instructed and uninstructed conditions separately. We then correlated these measures to examine the relationship between awareness of cue utility and the PVE. We found no correlation between the change in participant estimates and the change in the magnitude of cueing effects as a function of percentage valid, regardless of whether the participants were instructed, $r=.11, p=.511$, or not instructed, $r=-.05$, $p=.758$. The correlations are plotted in Fig. 5 .

\section{Discussion}

In Experiment2, the magnitude of the cueing effects increased as the percentage of valid trials increased, mirroring the PVEs in Experiment 1. Similarly, participants underestimated the actual percentage valid in the $75 \%$-valid condition, and overestimated it in the $25 \%$-valid condition, but accurately estimated the actual percentage valid in the $50 \%$-valid condition. 
E2: Central Arrow Cues $(\mathrm{N}=80)$

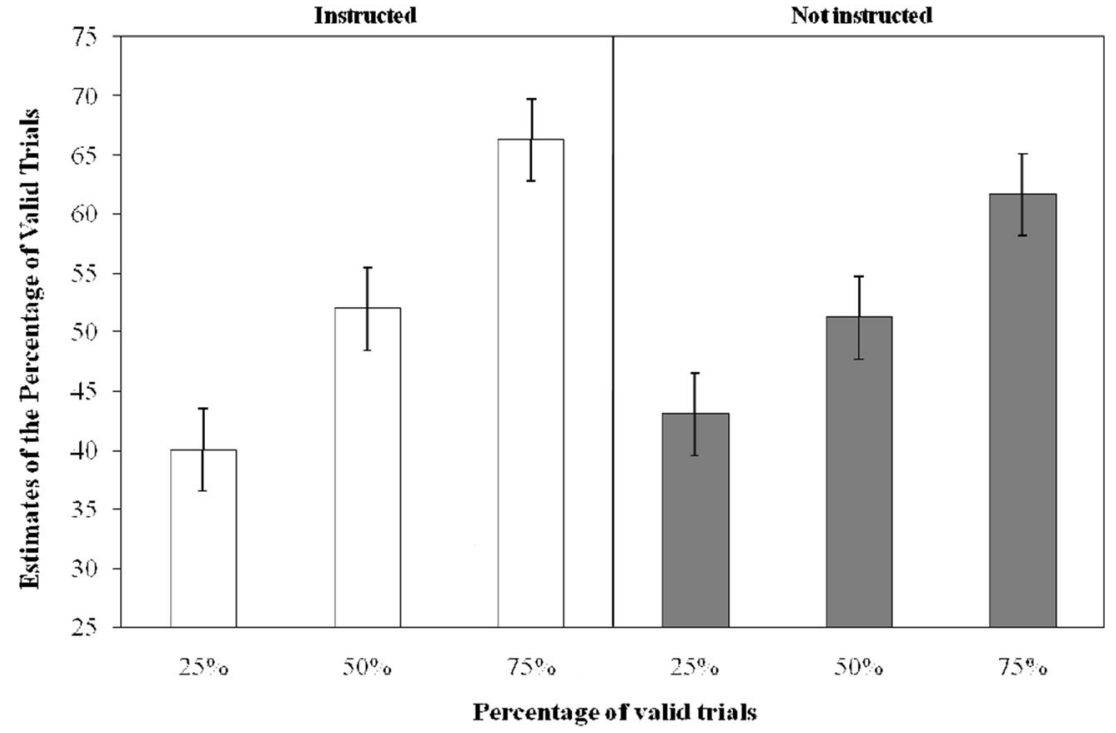

Fig. 4 Participant estimates of the percentages of valid trials as a function of instruction (instructed vs. not instructed) and percentage valid ( $25 \%$ vs. $50 \%$ vs. 75\%) in Experiment2. Error bars represent 95\% confidence intervals, as defined by Masson and Loftus (2003)

Also convergent with Experiment1, whether or not participants were instructed to attend to the cue-target relationship had no influence on their estimates of the percentage of valid trials. Furthermore, participants' specific estimates of cue validity and the validity effect itself were not significantly correlated. There was one minor exception to this result in the $25 \%$-valid instructed condition, but because the overall cueing effect was $2 \mathrm{~ms}$ in this condition and there was no significant relationship as the percentage of valid trials increased - and with it the PVE - this effect is of little consequence. Finally, we again found that the relative change in participants' estimates had no impact on the PVE. Taken together, these data suggest that the emergence of the PVE is not dependent on either a precise or a general level of awareness of the cuetarget validity relationship. This holds true regardless of whether or not participants are instructed to attend to the cue-target relationship. The finding that the PVE cannot be taken to reflect explicit volitional orienting, even for central cues, is convergent with Risko and Stolz (2010b).

Interestingly, although the instructions did not affect the relationship between the PVE and participant estimates (whether precise or general), we did find that the PVE was modulated by instructions. Specifically, the PVE was larger when participants were instructed to attend to the cue-target

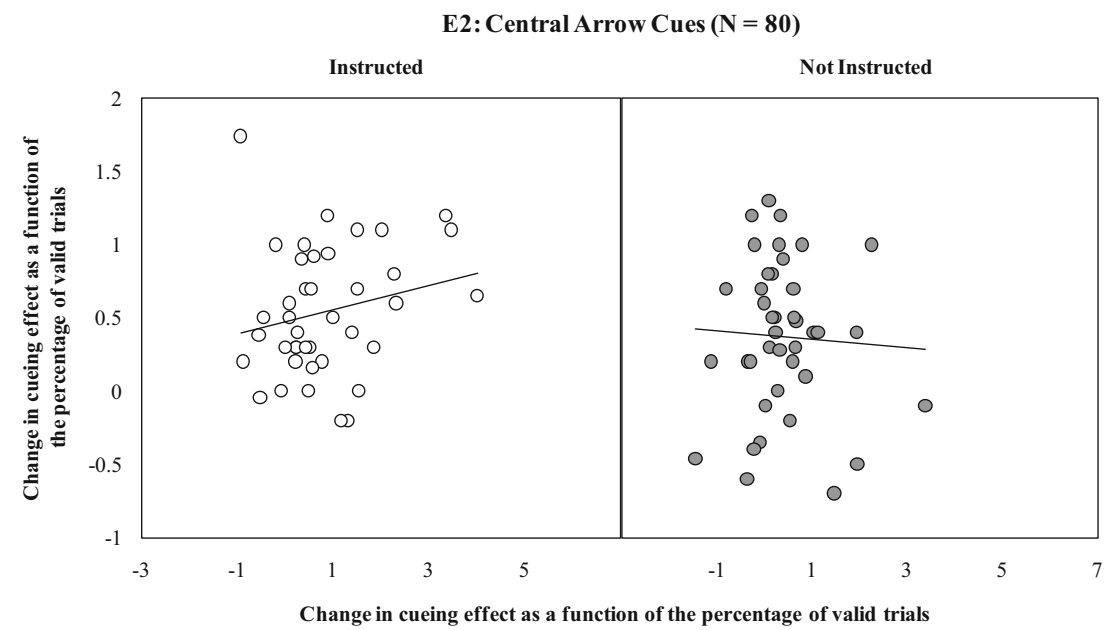

Fig. 5 The correlation between the change in participants' estimates (percentages) and the change in the magnitude of their cueing effects (in milliseconds) as a function of the percentage of valid trials, for each instruction condition (instructed vs. not instructed) in Experiment 1 
relationship than when they were not so instructed. This lends support to the idea that a volitional component can influence the nature of the PVE. Moreover, providing instructions has an effect on the PVE for central cues (Exp.2), but not for peripheral cues (Exp.1). The implication of this finding is that the orienting of attention to central and peripheral cues may be qualitatively different, and that the PVE for central cues may reflect volitional orienting, as was suggested by Bartolomeo and colleagues (2007).

\section{General discussion}

Allocating attention to a target item enhances its processing efficiency. Because attention is capacity-limited, allocating it to a location where a target is likely to appear results in shorter RTs to targets that appear at an attended/valid location, at the cost of longer RTs to targets at an unattended/unlikely target location. As the likelihood of a target appearing at a particular location increases, so does the allocation of attention to that location, and with it, the difference in RTs for targets at valid versus invalid locations. This change in RTs as a function of cue-target validity is known as the proportion validity cueing effect, and historically it was thought to reflect explicit changes in the control of attentional allocation-for example, strategic decisions by a participant to commit attention to a location that is likely to receive a target (Bartolomeo \& Chokron, 2002; Castel, Chasteen, Scialfa, \& Pratt, 2003; Danckert, Maruff, Crowe, \& Currie, 1998: Enns \& Brodeur, 1989; Jonides, 1981; Kingstone, 1992; Posner, 1980). It has been noted, however, that implicit associations between cue and target locations can also generate a PVE (Bartolomeo et al., 2007; Lambert \& Holmes, 2004; Lambert et al., 1999; Lambert \& Sumich, 1996; Risko \& Stolz, 2010b).

The question that we sought to examine in the present study was whether the PVEs generated by peripheral and central cues reflect explicit or implicit attentional control. In a series of studies, Bartolomeo and colleagues (2007) and Risko and Stolz (2010b) addressed this issue and converged on the conclusion that peripheral cues reflect implicit processes. Where they diverged was on the PVE generated in response to central cues. Bartolomeo et al.'s data suggested that the PVE was explicit in nature, whereas the Risko and Stolz (2010b) data suggested implicit control.

We noted that the lack of convergence may have derived from methodological differences between the studies, the most critical of which was how explicit awareness of cuetarget validity proportions was assessed. Risko and Stolz (2010b) required participants to generate a specific estimate of the cue-target contingency, whereas Bartolomeo et al. (2007) required participants merely to indicate whether or not the cue-target contingency was generally reliable. Our working hypothesis was that the PVE for central cues might be sensitive to a coarse level of explicit cue-target contingency awareness, but insensitive to awareness that is measured at a fine level. To test this issue, we modified the Risko and Stolz (2010b) paradigm to allow the measurement of both levels of awareness. In addition, we instructed half of our participants to attend to the cue-target relation, in order to examine whether the PVE was affected by the explicit engagement of attention.

Our findings were unequivocal. We replicated the finding from Bartolomeo et al. (2007) and Risko and Stolz (2010b) that the PVE for peripheral cues reflects implicit processing. Regardless of whether or not participants were instructed to attend to the cue-target relationship, neither the PVE nor the estimates of the percentages of valid trials were influenced. Furthermore, participants' specific estimates of cue validity and the validity effect itself were not related to one another. As such, our study provides strong and converging evidence for previous findings that the PVE for peripheral cues reflects implicit processing (Bartolomeo et al., 2007; Risko \& Stolz, 2010b).

The result for central cues was also clear, although slightly more complex. We found that the PVE does not require explicit awareness of cue-target contingency, as was measured by Risko and Stolz (2010b), nor a general cue-target awareness, as was measured by Bartolomeo et al. (2007). As with peripheral cues, instructing participants to attend or not to attend to the cue-target relationship had no influence on their estimates of the percentage of valid trials. Furthermore, participants' specific estimates of cue validity and the validity effect itself were not significantly correlated. These data are consistent with Risko and Stolz's (2010b) finding that the PVE generated with central cues reflects implicit processing. However, in support of Bartolomeo et al. (2007), we found that the explicit instruction to attend to the cue-target relation did affect, and enhance, the PVE. Therefore, a PVE for central cues cannot be assumed to reflect either implicit or explicit processes alone, but rather it is reasonable to conclude that the PVE for central cues may reflect a combination of the two processes. Whether this conclusion holds for central cues that do not elicit automatic orienting (Ristic \& Kingstone, 2012; Olk et al., 2014) is very much an open question, and one that we hope will be the subject of future investigation (we are grateful to an anonymous reviewer for raising this point).

In sum, the present data and those from previous investigations converge on the conclusion that the PVEs for both peripheral and central cues reflect implicit processing but, unlike the PVE for peripheral cues, the PVE for central cues is sensitive to explicit attentional modulation. The facts that awareness of cue-target contingencies is not required to produce the PVE, but that this awareness can modulate the PVE observed with central cues, may help to clarify the source of disagreement between Risko and Stolz (2010b) and Bartolomeo and colleagues (2007). 
Author note This work was supported by a Natural Sciences and Engineering Research Council (NSERC) of Canada graduate scholarship and by a Michael Smith Foundation of Health Research Trainee Award to S.N.L., as well as by a Killam Fellowship to C.S.C. and an NSERC Discovery Grant to A.K.

\section{References}

Bartolomeo, P., \& Chokron, S. (2002). Orienting of attention in left unilateral neglect. Neuroscience \& Biobehavioral Reviews, 26, 217 234.

Bartolomeo, P., Decaix, C., \& Siéroff, E. (2007). The phenomenology of endogenous orienting. Consciousness and Cognition, 16, 144-161. doi:10.1016/j.concog.2005.09.002

Castel, A. D., Chasteen, A. L., Scialfa, C. T., \& Pratt, J. (2003). Adult age differences in time course of inhibition of return. Journals of Gerontology, 58B, 256-P259.

Chica, A. B., \& Bartolomeo, P. (2010). Unconscious strategies? Commentary on Risko and Stolz, "The proportion valid effect in covert orienting: Strategic control or implicit learning?". Consciousness and Cognition, 19, 443-444. doi:10.1016/j.concog. 2009.09.010

Chica, A. B., Bartolomeo, P., \& Lupiáñez, J. (2013). Two cognitive and neural systems for endogenous and exogenous spatial attention. Behavioural Brain Research, 237, 107-123. doi:10.1016/j.bbr. 2012.09.027

Chun, M. M., \& Jiang, Y. (1998). Contextual cueing, Implicit learning and memory of visual context guides spatial attention. Cognitive Psychology, 36, 28-71. doi:10.1006/cogp.1998.0681

Chun, M. M., \& Jiang, Y. (1999). Top-down attentional guidance based on implicit learning of visual covariation. Psychological Science, 10, 360-365. doi:10.1111/1467-9280.00168

Cleeremans, A., Destrebecqz, A., \& Boyer, M. (1998). Implicit learning, news from the front. Trends in Cognitive Sciences, 2, 406-416.

Danckert, J., Maruff, P., Crowe, S., \& Currie, J. (1998). Inhibitory processes in covert orienting in patients with Alzheimer's disease. Neuropsychology, 12, 225-241.

Enns, J. T., \& Brodeur, D. A. (1989). A developmental study of covert orienting to peripheral visual cues. Journal of Experimental Child Psychology, 48, 171-189.

Funes, M. J., Lupiáñez, J., \& Milliken, B. (2007). Separate mechanisms recruited by exogenous and endogenous spatial cues: evidence from a spatial Stroop paradigm. Journal of Experimental Psychology: Human Perception \& Performance, 33, 348-362. doi:10.1037/ 0096-1523.33.2.348

Jonides, J. (1981). Voluntary versus automatic control over the mind's eye's movement. In J. Long \& A. Baddeley (Eds.), Attention and performance XI (pp. 187-203). Hillsdale: Erlbaum.

Kentridge, R. W., Heywood, C. A., \& Weiskrantz, L. (1999). Attention without awareness in blindsight. Proceedings of the Royal Society B, 266, 1805-1811.

Kingstone, A. (1992). Combining expectancies. Quarterly Journal of Experimental Psychology, 44A, 69-104. doi:10.1080/ 14640749208401284

Lambert, A. J., \& Holmes, J. (2004). Ageing and visual orienting in response to complex spatial cues. Brain Impairment, 5, 117-125.

Lambert, A., Naikar, N., McLachlan, K., \& Aitken, V. (1999). A new component of visual orienting: Implicit effects of peripheral information and subthreshold cues on covert awareness. Journal of Experimental Psychology: Human Perception and Performance, 25, 321-340. doi:10.1037/0096-1523.25.2.321
Lambert, A. J., \& Sumich, A. L. (1996). Spatial orienting controlled without awareness, a semantically based implicit learning effect. Quarterly Journal of Experimental Psychology, 49, 490-518.

López-Ramón, M. F., Chica, A. B., Bartolomeo, P., \& Lupiáñez, J. (2011). Attentional orienting and awareness: Evidence from a discrimination task. Consciousness and Cognition, 20, 745-755. doi: 10.1016/j.concog.2010.10.024

Masson, M. E. J., \& Loftus, G. R. (2003). Using confidence intervals for graphically based data interpretation. Canadian Journal of Experimental Psychology, 57, 203-220. doi:10.1037/h0087426

McCormick, P. A. (1997). Orienting attention without awareness. Journal of Experimental Psychology: Human Perception and Performance, 23, 168-180. doi:10.1037/0096-1523.23.1.168

Olk, B., Tsankova, E., Petca, A. R., \& Wilhelm, A. F. (2014). Measuring effects of voluntary attention: A comparison among predictive arrow, colour, and number cues. Quarterly Journal of Experimental Psychology, 67, 2025-2041. doi:10.1080/17470218.2014.898670

Posner, M. I. (1980). Orienting of attention. Quarterly Journal of Experimental Psychology, 32, 3-25. doi:10.1080/ 00335558008248231

Risko, E. F., \& Stolz, J. A. (2010a). On the nature of cognitive control and endogenous orienting: A response to Chica and Bartolomeo (2010). Consciousness and Cognition, 19, 445-446. doi:10.1016/j.concog. 2009.11.005

Risko, E. F., \& Stolz, J. A. (2010b). The proportion valid effect in covert orienting: Strategic control or implicit learning? Consciousness and Cognition, 19, 432-442. doi:10.1016/j. concog.2009.07.013

Ristic, J., \& Kingstone, A. (2012). A new form of human spatial attention: Automated symbolic orienting. Visual Cognition, 20, 244-264. doi: 10.1080/13506285.2012.658101

Shin, M. J., Marrett, N., \& Lambert, A. J. (2011). Visual orienting in response to attentional cues: Spatial correspondence is critical, conscious awareness is not. Visual Cognition, 19, 730-761.

Taylor, T. L., \& Klein, R. M. (1998). On the causes and effects of inhibition of return. Psychonomic Bulletin \& Review, 5, 625-643. doi:10. 3758/BF03208839

Theeuwes, J. (1991). Cross-dimensional perceptual selectivity. Perception \& Psychophysics, 50, 184-193. doi:10.3758/ BF03212219

Tipper, C., \& Kingstone, A. (2005). Is inhibition of return a reflexive effect? Cognition, 97, B55-B62. doi:10.1016/j.cognition.2005.02. 003

Tipples, J. (2008). Orienting to counterpredictive gaze and arrow cues. Perception \& Psychophysics, 70, 77-87. doi:10.3758/ PP.70.1.77

Van Selst, M., \& Jolicœur, P. (1994). A solution to the effect of sample size on outlier elimination. Quarterly Journal of Experimental Psychology, 47A, 631-650. doi:10.1080/14640749408401131

Vaquero, J. M., Fiacconi, C., \& Milliken, B. (2010). Attention, awareness of contingencies, and control in spatial localization: A qualitative difference approach. Journal of Experimental Psychology: Human Perception and Performance, 36, 1342-1357. doi:10.1037/ a0020406

Vecera, S. P., \& Rizzo, M. (2006). Eye gaze does not produce reflexive shifts of attention: Evidence from frontal-lobe damage. Neuropsychologia, 44, 150-159. doi:10.1016/j.neuropsychologia. 2005.04.010

Yantis, S., \& Jonides, J. (1990). Abrupt visual onsets and selective attention: Voluntary versus automatic allocation. Journal of Experimental Psychology: Human Perception and Performance, 16, 121-134. doi:10.1037/0096-1523.16.1.121 